\title{
Association Trends Between Antihypertensive Drug Therapies and Diastolic Hypotension in Emirati Patients with Type 2 Diabetes: A Single-Center Retrospective Longitudinal Study
}

\author{
Bashair M. Mussa - Rifat A. Hamoudi - Salah E. Abusnana
}

Received: May 18, 2018 / Published online: July 24, 2018

(C) The Author(s) 2018 present retrospective study. The main objectives of the present study were (1) to determine the prevalence of low diastolic blood pressure (DBP) and diastolic hypotension in this group of patients and (2) to investigate the associations, if any, between the use of various antihypertensive medications and low DBP and diastolic hypotension.

Results: The results of the present study showed that low DBP $(<70 \mathrm{mmHg})$ was experienced by $40 \%$ of the hypertensive type $2 \mathrm{DM}$ patients, whereas diastolic hypotension $(<60 \mathrm{mmHg})$ was reported to occur in about $10 \%$ of the patients. Another important factor that has been significantly correlated with diastolic hypotension is age $(p<0.01)$. Association trends have been reported between low DBP and diastolic hypotension and several antihypertensive therapies, including (1) monotherapies such as angiotensin-converting enzyme (ACE) inhibitors and angiotensin II receptor blockers (ARBs), (2) dual therapies such as ACE inhibitors in combination with thiazidelike diuretics (THLDs) or beta blockers, and (3) triple therapy combinations of ACE inhibitors with THLDs and potassium-sparing diuretics.

Conclusion: The use of antihypertensive medications, in particular ACE inhibitors and ARBs, appears to be a risk factor for the development of low DBP and diastolic hypotension in obese hypertensive Emirati patients with type 2 DM, whereas calcium channel blockers seem to be a safer option for this group of patients. 
Keywords: Angiotensin II receptor blocker; Angiotensin-converting enzyme inhibitors; Antihypertensive medications; Calcium channel blockers; Diastolic blood pressure; Diastolic hypotension; Systolic blood pressure; Type 2 diabetes

\section{INTRODUCTION}

The challenge of treating hypertension in obese patients with type 2 diabetes mellitus (T2DM) has revolutionized the classic concept of metabolic syndrome and redefined the relationship between its major elements: obesity, insulin resistance, and hypertension [1].

Hypertension is one of the major risk factors for morbidity and mortality in the general population, particularly in obese patients with T2DM. The latter have a double chance of developing hypertension compared to nondiabetic subjects with normal weight $[2,3]$. Most (75\%) T2DM patients die from cardiovascular complications associated with hypertension $[4,5]$. In addition, well-documented evidence has shown that obesity is a global epidemic and is strongly related to the development of hypertension and T2DM, emphasizing the interaction between the components of metabolic syndrome [6-9]. In good agreement with corresponding international data, previous studies performed in the Gulf region have shown that there is a high prevalence of hypertension among T2DM patients [10, 11]. Similarly, studies carried out in the United Arab Emirates (UAE) have reported high prevalences of DM, hypertension, and obesity in Emirati and non-Emirati populations [12, 13]. The UAE has experienced pronounced economic growth during the past few decades, which has led to increased prevalences of several risk factors for DM, obesity, and hypertension.

Classically, the main therapeutic path to controlling hypertension has been to decrease the systolic blood pressure (SBP) $(<140 \mathrm{mmHg})$ [14]. More recent findings have challenged this concept by demonstrating that a low diastolic pressure (DBP) is strongly associated with cardiovascular events, particularly myocardial infarction [15]. The risk of the latter in patients with a DBP of $60 \mathrm{mmHg}$ was found to be double that in patients with a DBP of $100 \mathrm{mmHg}$ [16]. These findings raised concerns regarding the effects of tight SBP control and simultaneous low DBP on cardiovascular outcomes. This leads to a very important question about the optimal level of SBP and whether it can be reached without increasing the incidence of diastolic hypotension. The latter is defined by several associations, including the American College of Cardiology, as a DBP of $<60 \mathrm{mmHg}[2,3]$.

It is noteworthy that patients with DM and hypertension have higher SBPs $(2-3 \mathrm{mmHg})$ and lower DBPs $(1-3 \mathrm{mmHg})$ than nondiabetic hypertensive patients. These findings, along with the outcomes of the Hypertension Optimal Treatment Study, have led to the development of new therapeutic targets $(<130 / 80 \mathrm{mmHg})$ for treating hypertension in patients with $\mathrm{DM}$ [17-19].

Therapy for hypertension in T2DM classically includes several subclasses of antihypertensive medications, and data comparing the outcomes of using each subclass are limited. However, in general, it is evident that angiotensin-converting enzyme (ACE) inhibitors, angiotensin receptor blockers (ARBs), diuretics, and beta blockers are superior to placebo. Importantly, it has been shown that the application of multiple combinations of these subclasses is strongly associated with a reduction in the SBP [14].

Although diastolic hypotension in DM patients is regarded as a major concern, the effects of multiple antihypertensive medications on the DBP are yet to be identified and reported. The literature lacks exhaustive studies of the relationship between the incidence of diastolic hypotension and the use of antihypertensive medications, and the susceptibility of obese patients with T2DM to diastolic hypertension development. Taking into account all of these findings and the points made above, the study reported in the present paper was designed (1) to determine the prevalence of diastolic hypotension in a cohort of T2DM patients with hypertension and (2) to investigate the associations, if any, between the 
use of antihypertensive medications, and low DBP and/or diastolic hypotension.

\section{METHODS}

\section{Data Source}

The present work focuses on a single-center retrospective longitudinal study conducted between June 2016 and June 2017 at Rashid Centre of Diabetes and Research (RCDR, Sheikh Khalifa Medical City, Ajman, UAE). This article is based on data that were extracted from electronic medical records and previously conducted studies, and does not report any interventional studies of human participants or animals that were performed by any of the authors.

All data were collected from an electronic medical records system (Diamond Database System). The following demographic, clinical, and medication data were collected from the medical records: age, age at diagnosis, gender, diabetes duration, SBP, DBP, pulse, body mass index (BMI), and hemoglobin A1c (HA1c). The analysis also included the lipid profile: total cholesterol, high-density lipoprotein (HDL), low-density lipoprotein (LDL), triglycerides, and antihypertensive medications.

Three thousand medical records of Emirati patients with DM were reviewed, and 500 medical records were randomly selected and screened. Those medical records were created by consultant diabetologists and endocrinologists who assessed the patients regularly. All patients with DM attended the diabetes clinics in RCDR on a quarterly basis (an initial visit and three follow-up visits per annum).

\section{Study Population}

Emirati patients aged between 18 and 70 years old with T2DM and hypertension were included in the present study, and all patients visited the diabetes clinic at least four times per annum. Patients with type $1 \mathrm{DM}$ and/or normal blood pressure were excluded. Four hundred and eight medical records were retrieved and included in the study based on the inclusion and exclusion criteria. The same group of patients were followed up after the initial visit (visit 1, V1) at visit 2 (6-month follow-up, V2), visit 3 (12month follow-up, V3), and visit 4 (18-month follow-up, V4).

\section{Outcomes Analysis}

The primary aims of the study were to investigate the presence of diastolic hypotension in Emirati patients with hypertension and T2DM and identify the relationships, if any, between a low DBP or diastolic hypotension and the use of various antihypertensive medications. The DBP was categorized into four groups: normal DBP (> $74 \mathrm{mmHg}$ ), low DBP (74-64 mmHg), diastolic hypotension $(63-54 \mathrm{mmHg})$, and severe diastolic hypotension $(<54 \mathrm{mmHg})$. These groups were sample-based and statistically derived using a mean DBP of $74 \mathrm{mmHg}$ as a reference point [1].

\section{Statistical Analysis}

All data are expressed as the mean $( \pm$ SD) and the number of patients (percentage) for continuous and discrete variables, respectively. An assessment of the normality of the data was conducted and a normal DBP distribution was observed using a Q-Q plot. In addition, the chisquare test and cross-tabulation were used to investigate the relationships, if any, between DBP and various antihypertensive medications. Two-tailed $p$ values of less than 0.05 were considered statistically significant. SPSS version 21.0 was used to carry out the statistical analysis.

\section{RESULTS}

A total of 500 patients were randomly selected between June 2016 and June 2017, and 92 patients were excluded based on the criteria set prior to the screening procedure. Four hundred and eight Emirati patients with T2DM and hypertension were included in the study. Those 
patients visited RCDR diabetes clinic at least four times: V1-V4.

As shown in Table 1, the average age of the studied population was $61.0 \pm 10.0$ years, and the majority of the patients (72\%) were female. In addition, the average age at diabetes diagnosis was $46.2 \pm 10.9$ years, the average duration of the disease was about $14.7 \pm 7.5$ years, and the average initial HbA1c was $8.8 \pm 2.0 \%$. The studied population had uncontrolled DM, and the lowest $\mathrm{HbA1c}$ reported at V4 was $7.9 \pm 1.5 \%$.

Obesity was a common feature of this population, with an average BMI of $34.0 \pm 8.2$. A slight decrease in BMI was observed during the follow-up visits (V2, $33.8 \pm 7.8$; V3, $33.9 \pm 8.2$; $\mathrm{V} 4,33.6 \pm 7.8)$. The average lipid profile during the initial visit was as follows: total cholesterol $4.4 \pm 1.1$, triglycerides $1.6 \pm 0.9$, HDL $1.2 \pm 0.3$, and LDL $2.7 \pm 0.9$, and the average profile did not significantly change during V2, V3, and V4. SBP $(142.0 \pm 22.3 \mathrm{mmHg})$ was high during the initial visit but decreased with each subsequent visit, whereas the DBP was initially low $(74.0 \pm$ $12.34)$ and remained so at V2 (71.8 \pm 10.0$)$, V3 $(73.1 \pm 9.6)$, and V4 $(72.8 \pm 8.8)$; this decrease in DBP across visits was statistically significant $(p<0.05)$.

The results of the present study show that a low DBP $(<70 \mathrm{mmHg})$ was experienced by $40 \%$ of the hypertensive T2DM Emirati patients, while diastolic hypotension $(<60 \mathrm{mmHg})$ was reported for $10 \%$ of these patients. Age was found to be significantly correlated with diastolic hypotension $(p<0.01)$, and an inverse relationship between DBP and age was observed.

Different classes of antihypertensives were used as monotherapies and in combined therapies to control hypertension in this group of patients. As shown in Table 2, eight antihypertensive classes were used, including beta blockers, ACE inhibitors, ARBs, direct renin inhibitors (DRIs), calcium channel blockers (CCBs), and diuretics (loop diuretics, potassium-sparing diuretics (PSDs), and thiazide-like diuretics

Table 1 Demographic and clinical data for the studied population throughout the four clinical visits

\begin{tabular}{lllll}
\hline Variable & Visit 1 & Visit 2 & Visit 3 & Visit 4 \\
\hline Subjects $(n)$ & 408 & - & - & - \\
Age (years) & $61.0 \pm 10.0$ & - & - & - \\
Gender & & & \\
No. females, $n$ (\%) & $292(72)$ & - & - & - \\
Age at diagnosis (years) & $46.2 \pm 10.9$ & - & - & - \\
Diabetes duration (years) & $14.7 \pm 7.5$ & - & $33.9 \pm 8.2$ & - \\
BMI (kg/m ${ }^{2}$ ) & $34.0 \pm 8.2$ & $33.8 \pm 7.8$ & $8.0 \pm 1.7$ & $7.9 \pm 1.5$ \\
HbA1C (\%) & $8.8 \pm 2.0$ & $8.0 \pm 1.6$ & $4.1 \pm 1.0$ & $4.1 \pm 0.9$ \\
Total cholesterol (mmol/L) & $4.4 \pm 1.1$ & $4.1 \pm 0.9$ & $1.7 \pm 1.0$ & $1.7 \pm 1.0$ \\
Triglycerides (mmol/L) & $1.6 \pm 0.9$ & $1.6 \pm 1.1$ & $1.2 \pm 0.3$ & $1.1 \pm 0.3$ \\
HDL (mg/dL) (mmol/L) & $1.2 \pm 0.3$ & $1.1 \pm 0.3$ & $2.4 \pm 0.8$ & $2.4 \pm 0.8$ \\
LDL (mg/dL) (mmol/L) & $2.7 \pm 0.9$ & $2.4 \pm 0.8$ & $140.0 \pm 22.6$ & $140.9 \pm 19.6$ \\
Systolic blood pressure & $142.0 \pm 22.3$ & $137.4 \pm 19.6$ & $73.1 \pm 9.6^{*}$ & $72.8 \pm 8.8^{*}$ \\
Diastolic blood pressure & $74.0 \pm 12.3$ & $71.8 \pm 10.0^{*}$ & & - \\
\hline
\end{tabular}

$B M I$ body mass index, $H b A 1 C$ hemoglobin Alc, $H D L$ high-density lipoprotein, $L D L$ low-density lipoprotein ${ }^{*} p<0.05$ 
Table 2 Classes of antihypertensive medications used by the studied population

\begin{tabular}{ll}
\hline \multicolumn{2}{l}{ Antihypertensive medications } \\
\hline Class & Medication \\
\hline Beta blockers & Atenolol, bisoprolol carvedilol \\
$\begin{array}{l}\text { Angiotensin-converting } \\
\text { enzyme (ACE) }\end{array}$ & $\begin{array}{c}\text { Perindopril, lisinopril } \\
\text { enalapril, cilazapril } \\
\text { inhibitors }\end{array}$ \\
fosinopril \\
Angiotensin II receptor & $\begin{array}{c}\text { Indapamide, losartan } \\
\text { blocker (ARBs) }\end{array}$ \\
& $\begin{array}{l}\text { irbesartan, valsartan } \\
\text { telmisartan }\end{array}$ \\
Direct renin inhibitors & Aliskiren \\
(DRIs) & \\
Calcium channel blockers & Amlodipine, nifedipine \\
(CCBs) & lercanidipine, verapamil, \\
& felodipine \\
Potassium-sparing & Hydrochlorothiazide, \\
diuretics (PSDs) & spironolactone \\
Loop diuretics & Furosemide \\
Thiazide-like diuretics & Indapamide \\
(THLDs) & \\
\hline
\end{tabular}

(THLDs)). Aromatic L-amino acid decarboxylase inhibitors and imidazoline receptor agonists were rarely used as antihypertensive medications in the studied population, so they were excluded from the analysis.

As shown in Table 3 , the percentage of the total number of patients using antihypertensive monotherapy (V1, 39\%) decreased slightly across the visits (V2, 32\%; V3, 29\%; V4, 25\%), whereas dual antihypertensive medications appear to have been used by approximately the same percentage of patients across the visits (V1, 36\%; V2, 33\%, V3, 32\%; V4, 32\%). In contrast to the monotherapies, the use of triple antihypertensive medications increased slightly across the visits (V1, 24\%; V2, 33\%; V3, 38\%; V4, 43\%). The percentage of patients with normal DBP (> $70 \mathrm{mmHg}$ ) was comparable across visits (V1, 48\%; V2, 38\%; V3, 43\%; V4, 42\%); similarly, the percentage of patients with low
DBP (74-64 mmHg) and the percentage with diastolic hypotension $(63-54 \mathrm{mmHg})$ did not change significantly across the visits. Severe diastolic hypotension $(<54 \mathrm{mmHg})$ was rarely experienced by this group of patients: $3 \%$ was the highest percentage of patients with severe diastolic hypotension across the visits, as shown in Table 3. Therefore, severe diastolic hypotension $(<54 \mathrm{mmHg})$ was not included in our analysis of the association between antihypertensive medications subclasses and DBP. In addition, normal DBP (>70 $\mathrm{mmHg}$ ) was not included in the analysis of the subclasses of antihypertensive medications because this analysis focused on low DBP and diastolic hypotension.

The findings of the present study show that although eight subclasses of antihypertensive medications were used as monotherapies in this cohort, only four of those subclasses (ACE inhibitors, ARBs, beta blockers, and CCBs) were chosen as initial treatments for hypertension and used consistently throughout the follow-up period (Table 4). Loop diuretics and DRIs were also used as initial monotherapies but were alternated during the follow-up period. In contrast, PSDs were used as a monotherapy during the later visits. Association trends of ACE inhibitors, ARBs, and beta blockers with low DBP and diastolic hypotension were observed across the visits, and these association trends were particularly significant at V3 $(p<0.05)$ (Table 4$)$.

It has been observed that dual antihypertensive therapy of ACE inhibitors in combination with THLDs or CCBs or beta blockers has a strong tendency to cause a low DBP and diastolic hypotension, and this association was found to be very significant at V1 $(p<0.01)$ (Table 5). In addition, an association trend between low DBP and diastolic hypotension and ACE inhibitors combined with either ARBs or loop diuretics was reported. This trend was also observed for ACE inhibitors used in combination with PSDs, but was confined to the last visit only. Similarly, ARBs used in combination with THLDs or CCBs or beta blockers or loop diuretics appear to be significantly associated with a low DBP and diastolic hypotension (V1, $p<0.01$ ). As shown in Table 5 , an association trend between low DBP and ARBs in 


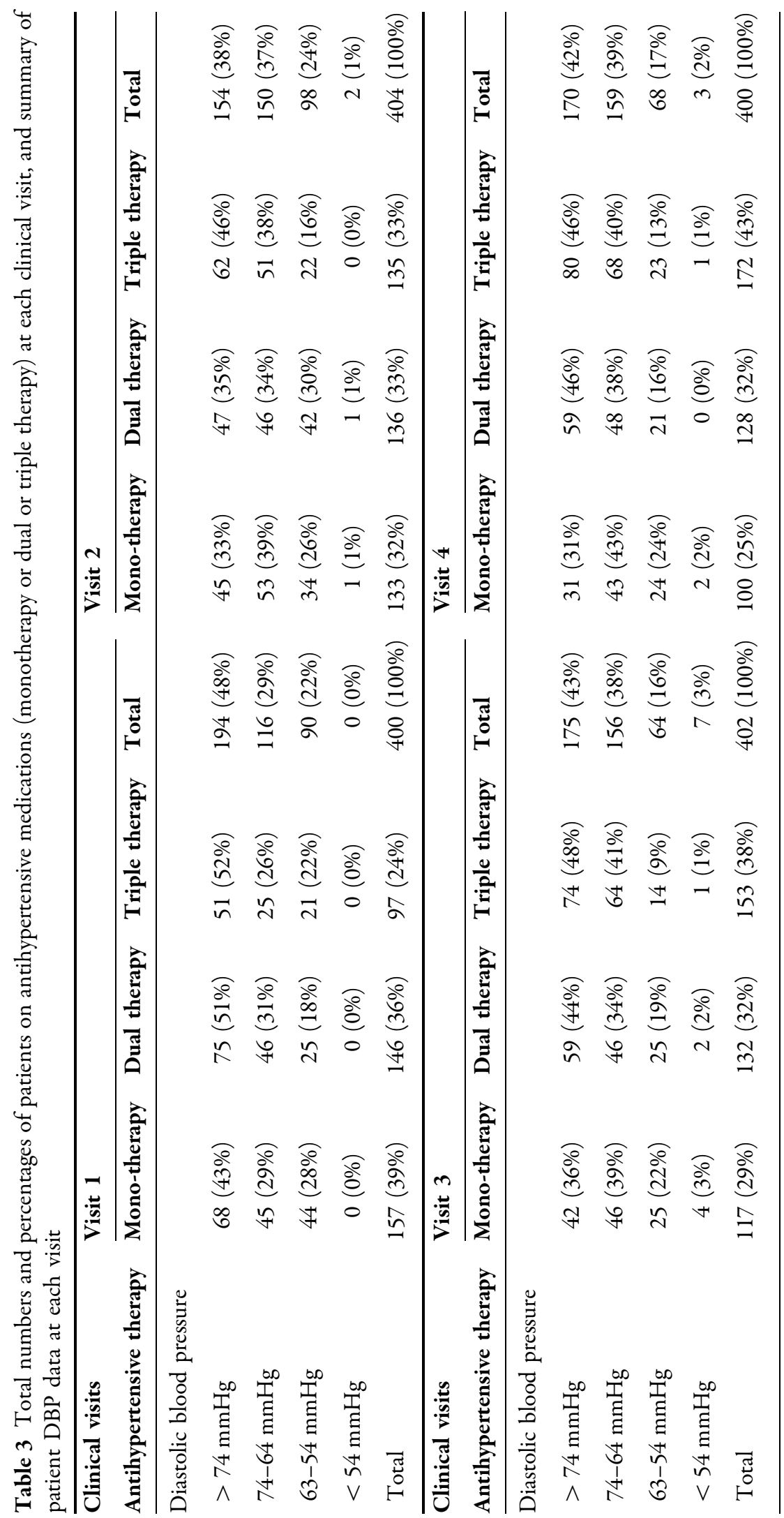




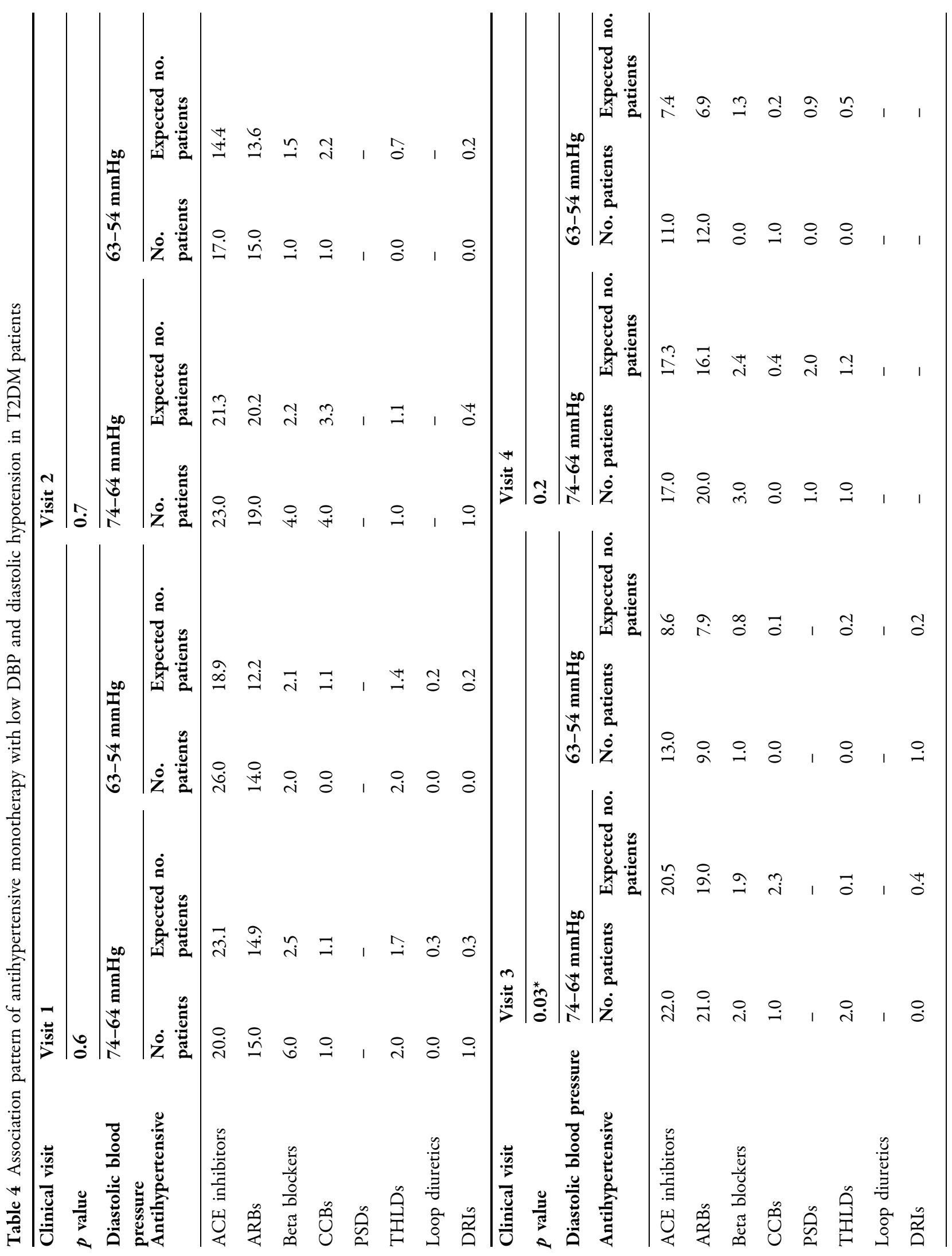




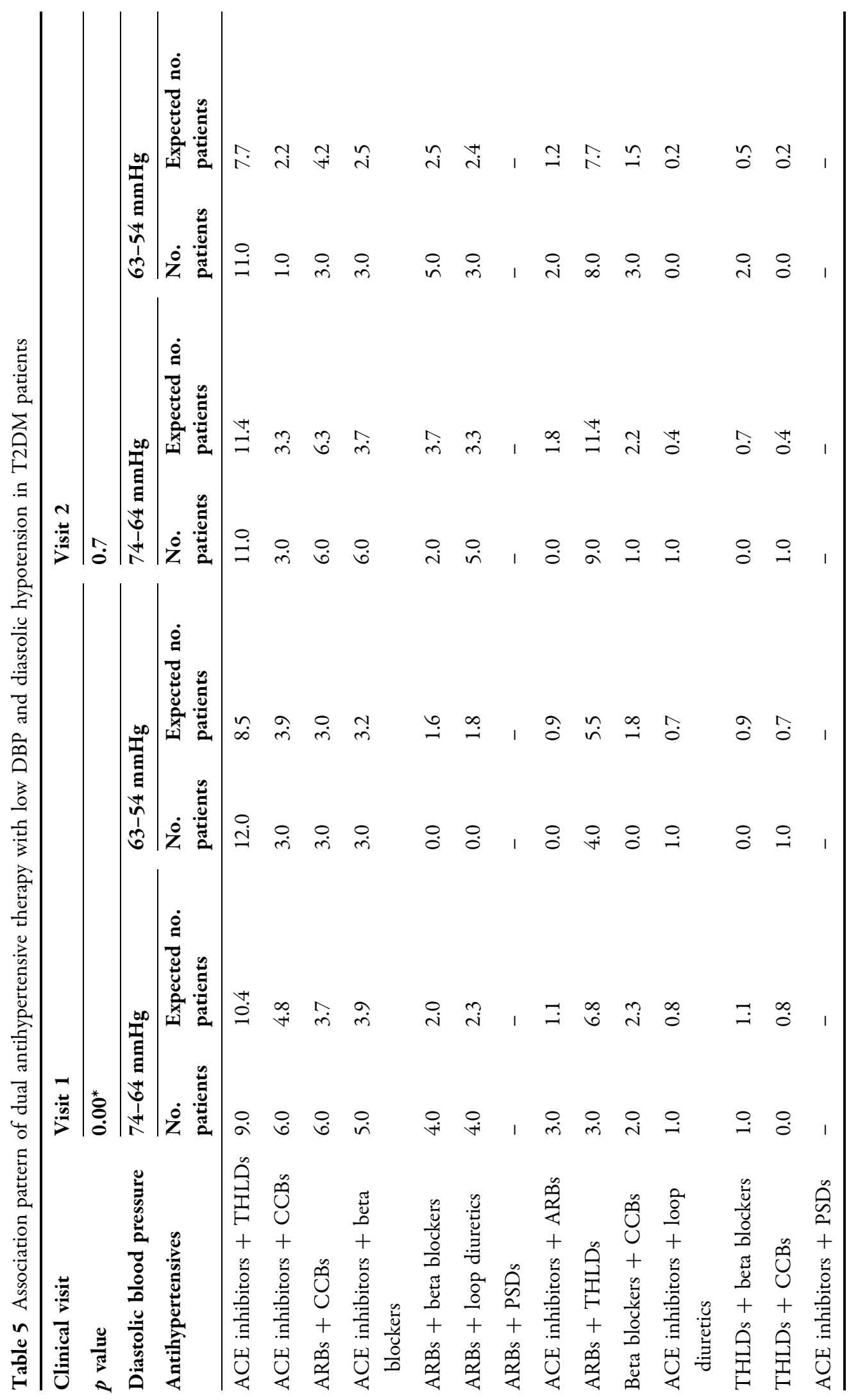




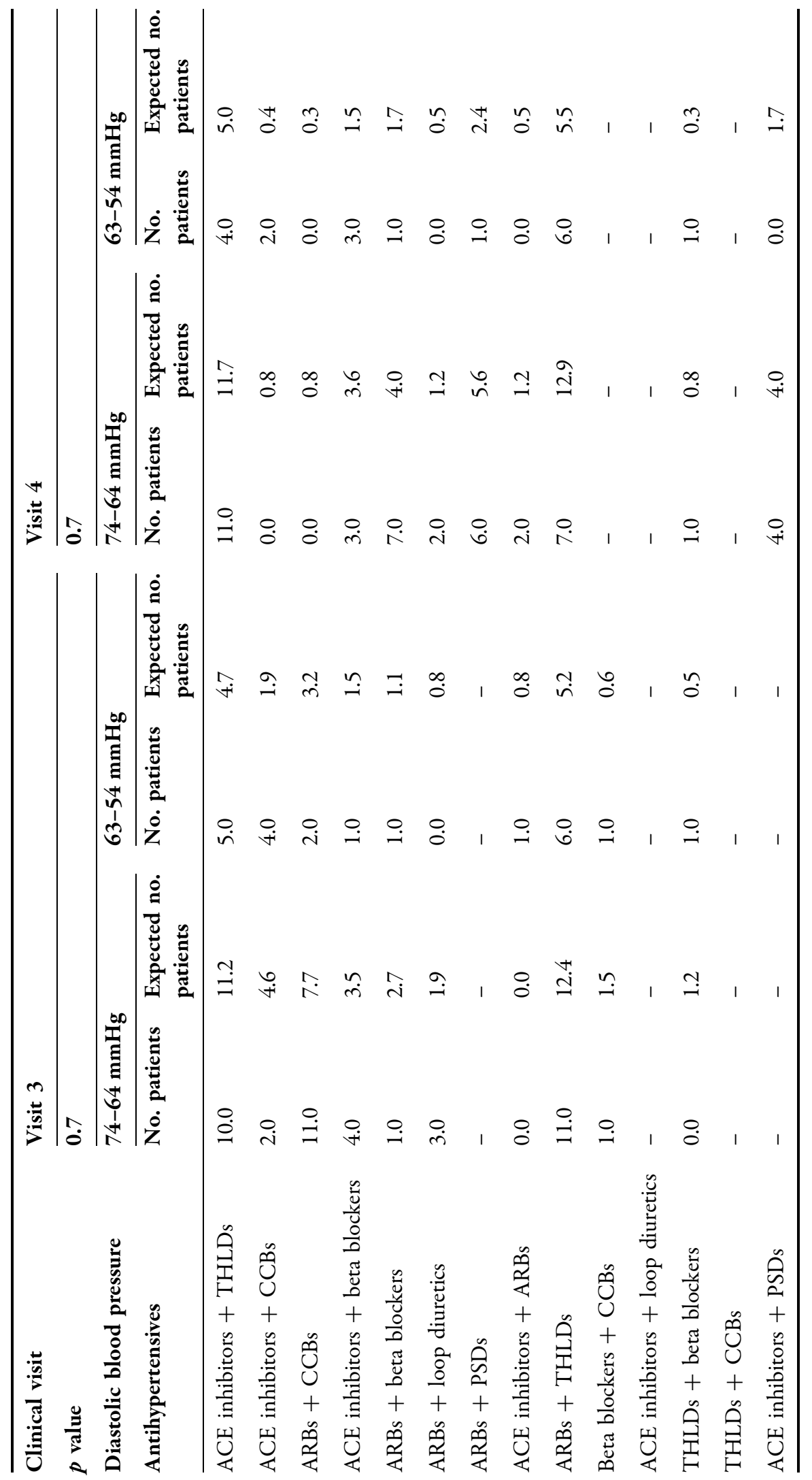




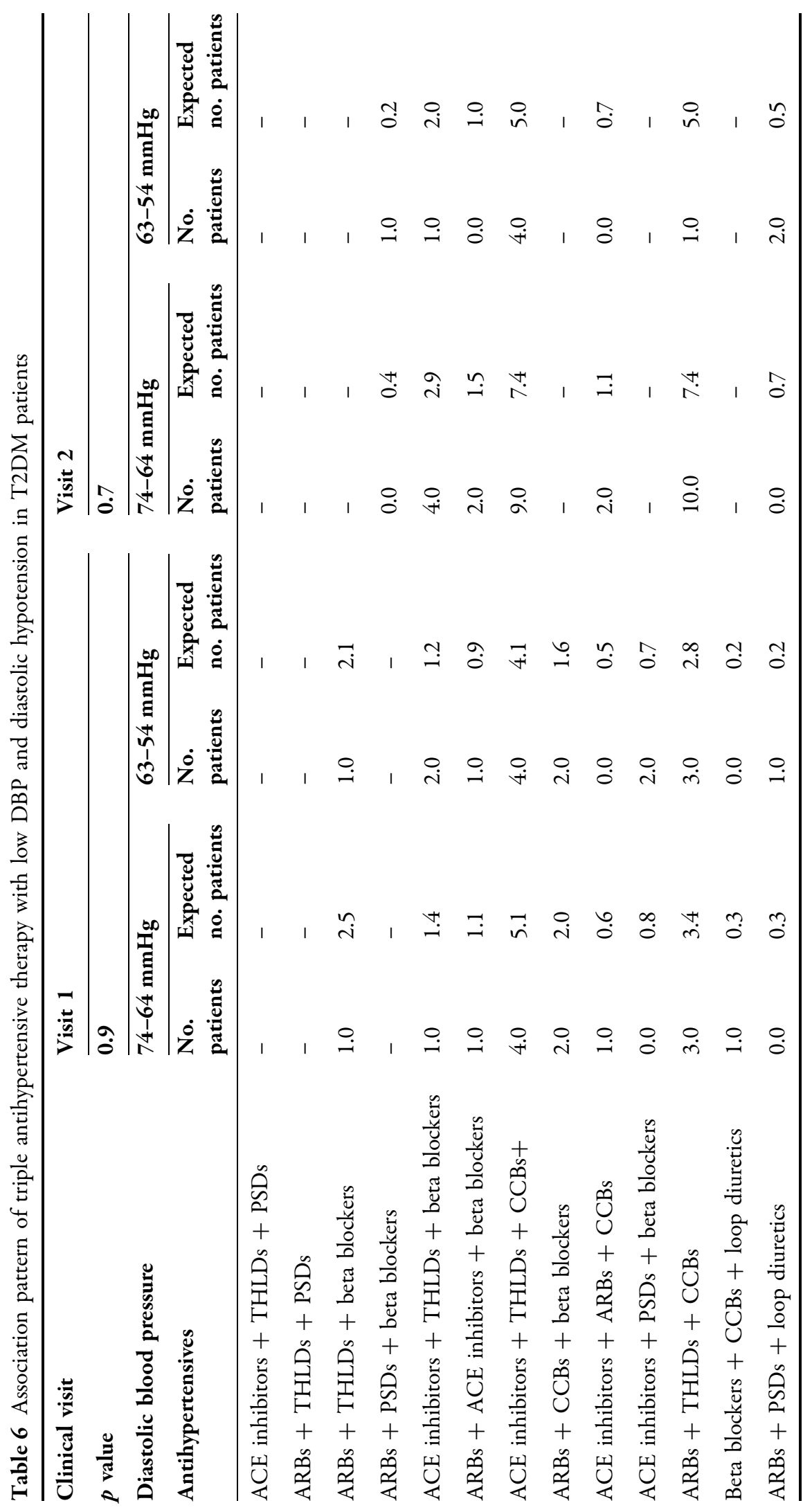




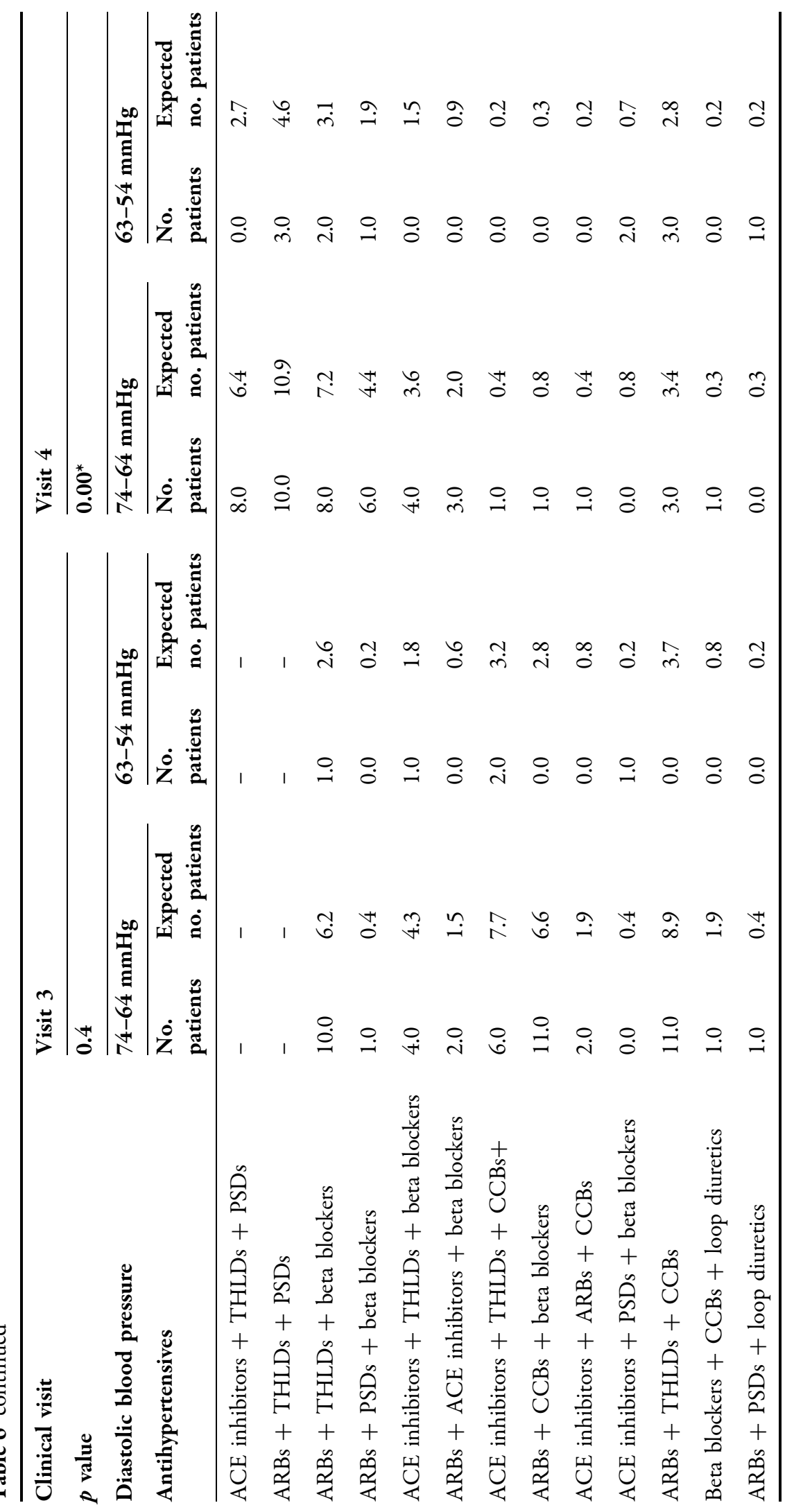


combination with PSDs was observed at the last visit. On the other hand, THLDs combined with either CCBs or beta blockers showed only a weak association with low DBP and diastolic hypotension across most of the visits.

Analysis of the associations of the triple antihypertensive medications with low DBP and diastolic hypotension was carried out, and a summary of the results is presented in Table 6. Those results reveal strong and significant associations (V4, $p<0.01$ ) of low DBP and diastolic hypotension with the following combinations of antihypertensive medications: (1) ACE inhibitors and THLDs and PSDs, (2) ARBs and THLDs and PSDs, and (3) ARBs and beta blockers and PSDs. This observation was confined to V4, but the combination of ARBs with THLDs and beta blockers showed an association trend at V3. In addition, THLDs used in combination with CCBs and either ACE inhibitors or ARBs showed a tendency to cause low DBP and diastolic hypotension (Table 6).

\section{DISCUSSION}

It is well documented that obesity, DM, and hypertension are key elements in the development of adverse cardiovascular outcomes [20]. Interestingly, a meta-analysis of some randomized controlled trials and observational studies questioned the involvement of obesity as a main factor in the enhanced mortality rate due to cardiovascular complications. This phenomenon is called the "obesity paradox" [21]. Follow-up studies have shown that prolonged and intensive use of medications following revascularization by percutaneous coronary intervention provides a reasonable explanation for the obesity paradox [22]. Although tight control of the SBP is crucial to avoiding or minimizing these adverse outcomes, various opinions have been reported regarding the potential link of constricted SBP with diastolic dysfunction [23, 24].

The principal finding of the present study is that antihypertensive therapies tended to lower the DBP and cause diastolic hypotension in obese Emirati patients with T2DM. In agreement with previous reports, the present study demonstrated that antihypertensive monotherapy treatment strategies include ACE inhibitors, ARBs, CCBs, beta blockers, and THLDs [25]. However, additional antihypertensive classes, such as loop diuretics, DRIs, and PSDs were also used as monotherapies to treat hypertension in this group of patients. It is noteworthy that PSDs were used as an alternative to classical antihypertensive options late in the treatment course. Compared to the other monotherapy treatments that were used, ACE inhibitors and ARBs showed significant associations with a low DBP and diastolic hypotension, suggesting that renin-angiotensin-aldosterone system blockade may have negative effects on diastolic function. In contrast, the latter was investigated in the Left Ventricular Mass and Diastolic Function (ELVERA) trial, and an improvement in diastolic function in response to lisinopril treatment was reported. It is important to note that this trial was conducted in hypertensive patients who had already been diagnosed with diastolic dysfunction prior to the trial [26]. Other trials have also reported that ACE inhibitors and ARBs produced reductions in vasoconstriction and cardiac volume load and therefore significantly improved diastolic hypotension. Those trials were conducted in different populations, including Swedish and Chinese, using different hypertensive subclasses. Conflicting data were subsequently published that led to doubt over the prognostic beneficial effects of ACE inhibitors and ARBs on diastolic function [27-29]. These doubts were supported by the findings of some trials conducted over long periods, including the Irbesartan in Heart Failure with Preserved Ejection Fraction trial (one-year follow-up) and the Candesartan in Heart Failure trial (three-year follow-up), which showed that neither irbesartan nor candesartan produced a benefit over placebo in cardiovascular outcomes [30, 31].

The study reported in this paper was a noninterventional study conducted in Emirati T2DM patients. None of those patients had previously been diagnosed with diastolic dysfunction, which may explain the difference in diastolic pressure outcomes between this study and the other studies. 
In good agreement with previous reports, the results of the present study fail to demonstrate any association of CCBs with low DBP or diastolic hypotension. It was previously found that CCBs help to maintain healthy diastolic function by attenuating calcium homeostasis, slowing the heart rate, and improving several diastolic parameters at the one- and two-year follow-up visits, suggesting superior prognostic effects to ACE inhibitors and ARBs [26, 32]. Similarly, the findings of the present study show that the use of diuretics (PSDs, THLDs, and loop diabetics) as monotherapies for short or long periods in the studied population did not produce a pronounced reduction in the DBP; this was also the case for DRIs. Although the effects of diuretics on hypertension have been studied in depth, very few reports have investigated the role of these antihypertensive agents in DBP. Those reports, along with the results of the present study, suggest that the use of diuretics in hypertensive patients does not adversely affect diastolic function [28, 33].

It has been found that a combined antihypertensive treatment is key to achieving optimal control of the BP and maintaining quality of life, and $75 \%$ of patients who achieved these targets were on combined therapies [34]. Another important finding of the present study is that dual therapy of ACE inhibitors with either THLDs or CCBs or beta blockers was significantly associated with a low DBP and diastolic hypotension. Although it seems that ACE inhibitors combined with THLDs produce similar effects to ACE inhibitors combined with beta blockers, other studies have demonstrated that the latter combination is more effective and has safer endpoints when used as an initial combination therapy [25]. In addition, the present study found that ACE inhibitors in combination with ARBs or diuretics (including PSDs or loop diuretics) were not associated with diastolic parameters.

Interestingly, a significant association pattern was observed with low DBP and diastolic hypotension when the antihypertensive treatment was a combination of ARBs with THLDs or CCBs. Furthermore, other combinations of ARBs with beta blockers and loop diuretics were significantly associated with a low DBP but not with diastolic hypotension. However, these combinations did show association trends with a low DBP and diastolic hypotension when used later in the treatment course.

Although the American Society of Hypertension identified a group of antihypertensive combinations (ACE inhibitors with beta blockers and ARBs with beta blockers) as being less effective at controlling high blood pressure [34], these combinations seemed to be effective at lowering the DBP in the present study population.

The evaluation of the effects of triple antihypertensive therapies on DBP performed in the present study demonstrated that ACE inhibitors or ARBs with diuretics (THLDs or PSDs) and beta blockers are significantly associated with a low DBP (74-64 mmHg). However, none of the triple antihypertensive combinations were associated with diastolic hypotension. This may lead to doubt over the finding that tight hypertension control using multiple antihypertensive medications always leads to diastolic hypotension in elderly patients. An alternative explanation is that the effects of antihypertensive therapies on diastolic function depend mainly on the medicine classes used, not the number of medications. This statement is supported by the results of the Avoiding Cardiovascular Events Through Combination Therapy in Patients Living With Systolic Hypertension Trial, which showed that triple therapy was more efficient at controlling BP and produced safer cardiovascular outcomes [35].

It is important to note that the main difference between the present study and previous studies is that the population studied in this work were obese patients with hypertension and T2DM. It has been shown that T2DM is one of the main factors that can lead to unexpected outcomes concerning the effects of antihypertensive therapies and diastolic function [36]. In addition, it has been observed that ethnicity influences how the antihypertensive medications affect DBP, so it is important to note the homogeneity of the population studied in the present work (all patients were Emiratis) [37].

In agreement with previous reports, the present study found that triple therapy with ARBs, ACE inhibitors, and CCBs had a good safety profile, and no correlation of this triple 
therapy with a low DBP or diastolic hypotension has been reported [38].

International guidelines for controlling hypertension have recommended targets, SPB/ DBP $<130 / 80 \mathrm{mmHg}$, for hypertensive patients with T2DM. These are $10 \mathrm{mmHg}$ lower than the targets for antihypertensive patients with normal glycemia (SPB/DBP $<140 / 90 \mathrm{mmHg}$ ) $[39,40]$. Taking into account the outcomes of the present study, it seems reasonable to utilize the same concept and propose a cutoff value for diastolic hypotension in patients with T2DM. In this group of patients, we propose that diastolic hypotension should be defined as a DBP of less than $70 \mathrm{mmHg}$, which is $10 \mathrm{mmHg}$ higher than the previously specified level $(60 \mathrm{mmHg})$.

One of the main limitations of the present study is the small sample size for each group. The small sample sizes occurred because only patients for whom the clinical parameters of interest were recorded at all visits were accepted. In addition, it was important to differentiate between low diastolic pressure and diastolic hypotension using different categories. This limitation could be overcome in the future by including a larger sample at the beginning of the study.

\section{CONCLUSION}

The present study investigated various subclasses of antihypertensive medications and their associations with low DBP and diastolic hypotension in T2DM patients for the first time. A significant association of monotherapy using ACE inhibitors or ARBs with low DBP or diastolic hypotension was observed. The same associative pattern was observed for dual antihypertensive therapies using ACE inhibitors and THLDs, CCBs, or beta blockers. Triple antihypertensive combinations including ACE inhibitors or ARBs plus diuretics (THLDs or PSDs) and beta blockers were significantly associated with low DBP but not with diastolic hypotension. These results provide new insights into how diastolic hypotension should be defined and aid the formulation of recommendations for obese Emirati patients with T2DM. Further investigations of the relationships between antidiabetic medications and diastolic hypotension should lead to a better understanding of this type of hypotension in T2DM patients.

\section{ACKNOWLEDGEMENTS}

The authors would like to acknowledge Dr. Noor Amer, Dr. Hessa Qatami, and Dr. Ahd ElNosh for their help with the collection and coding of study data.

Funding. No funding or sponsorship was received for this study or for the publication of this article. The article processing charges were funded by the authors.

Authorship. All named authors (Bashair M. Mussa, Rifat A. Hamoudi, and Salah E. Abusnana) meet the International Committee of Medical Journal Editors (ICMJE) criteria for authorship for this article, take responsibility for the integrity of the work as a whole, and have given their approval for this version to be published.

Disclosures. The authors of the present study (Bashair M. Mussa, Rifat A. Hamoudi, and Salah E. Abusnana) have no personal financial or nonfinancial conflicts of interest to disclose.

Compliance with Ethics Guidelines. This article is based on data that were extracted from electronic medical records and previously conducted studies, and does not contain any interventional studies of human participants or animals performed by any of the authors.

Data Availability. The research data used in the preparation of the manuscript are available from Dr. Bashair M. Mussa on reasonable request.

Open Access. This article is distributed under the terms of the Creative Commons Attribution-NonCommercial 4.0 International License (http://creativecommons.org/licenses/ by-nc/4.0/), which permits any noncommercial use, distribution, and reproduction in any medium, provided you give appropriate 
credit to the original author(s) and the source, provide a link to the Creative Commons license, and indicate if changes were made.

\section{REFERENCES}

1. Alberti K, Zimmet P, Shaw J, Group. IETFC. The metabolic syndrome-a new worldwide definition. Lancet. 2005;366(9491):1059-62.

2. Bild D, Teutsch SM. The control of hypertension in persons with diabetes: a public health approach. Public Health Rep. 1987;102(5):522-9.

3. Sowers JR, Epstein M, Frohlich ED. Diabetes, hypertension, and cardiovascular disease: an update. Hypertension. 2001;37(4):1053-9.

4. Campbell NR, Gilbert RE, Leiter LA, Larochelle P, Tobe S, Chockalingam A, et al. Diabetes, hypertension, and cardiovascular disease: an update. Can Fam Physician. 2011;57(9):997-1002.

5. Stirban A, Tschoepe D. Cardiovascular complications in diabetes: targets and interventions. Diabetes Care. 2008;Suppl 2:S215-21.

6. WHO. Obesity: preventing and managing the global epidemic. World Health Organ Tech Rep Ser. 2000;894(i-xii):1-253.

7. Wannamethee SG, Shaper AG. Weight change and duration of overweight and obesity in the incidence of type 2 diabetes. Diabetes Care. 1999;22(8):1266-72.

8. Gregg EW, Cheng YJ, Cadwell BL, Imperatore G, Williams DE, Flegal KM, et al. Secular trends in cardiovascular disease risk factors according to body mass index in US adults. JAMA. 2005;293(15):1868-74.

9. Mandal A. Study of prevalence of type 2 diabetes mellitus and hypertension in overweight and obese people. J Family Med Prim Care. 2014;3(1):25-8.

10. Alhyas L, McKay A, Balasanthiran A, Majeed A. Prevalence of overweight, obesity, hyperglycaemia, hypertension and dyslipidaemia in the Gulf: systematic review. JRSM Short Rep. 2011;2(7):55.

11. Aljefree N, Ahmed F. Prevalence of cardiovascular disease and associated risk factors among adult population in the Gulf region: a systematic review. Adv Public Health. 2015;2015:1-23.

12. Jelinek HF, Osman WM, Khandoker AH, Khalaf K, Lee $\mathrm{S}$, Almahmeed $\mathrm{W}$, et al. Clinical profiles, comorbidities and complications of type 2 diabetes mellitus in patients from United Arab Emirates. BMJ Open Diabetes Res Care. 2017;5(1):e000427.

13. Mussa B, Abduallah Y, Abusnana S. Prevalence of hypertension and obesity among emirati patients with type 2 diabetes. J Diabetes Metab. 2016;7(1):1-5.

14. American Diabetes Association. Treatment of hypertension in adults with diabetes. Diabetes Care. 2002;25(Suppl 1):s71-3.

15. Benetos A, Thomas F, Bean K, Gautier S, Smulyan H, Guize L. Prognostic value of systolic and diastolic blood pressure in treated hypertensive men. Arch Intern Med. 2002;162(5):577-81.

16. D'Agostino RB, Belanger AJ, Kannel WB, Cruickshank JM. Relation of low diastolic blood pressure to coronary heart disease death in presence of myocardial infarction: the Framingham Study. BMJ. 1991;303:385-9.

17. Estacio RO, Jeffers BW, Gifford N, Schrier RW. Effect of blood pressure control on diabetic microvascular complications in patients with hypertension and type 2 diabetes. Diabetes Care. 2000;Suppl 2:B54-64.

18. Hansson L, Zanchetti A, Carruthers SG, Dahlöf B, Elmfeldt D, Julius S, et al. Effects of intensive bloodpressure lowering and low-dose aspirin in patients with hypertension: principal results of the Hypertension Optimal Treatment (HOT) randomised trial. HOT Study Group. Lancet. 1998;351(9118):1755-62.

19. UK Prospective Diabetes Study Group. Tight blood pressure control and risk of macrovascular and microvascular complications in Type 2 diabetes. $\mathrm{Br}$ Med J. 1998;317:703-13.

20. Rosendorff C, Lackland DT, Allison M, Aronow WS, Black HR, Blumenthal RS, et al. Treatment of hypertension in patients with coronary artery disease: a scientific statement from the American Heart Association, American College of Cardiology, and American Society of Hypertension. J Am Soc Hypertens. 2015;9(6):453-98.

21. Bundhun PK, Li N, Chen MH. Does an obesity paradox really exist after cardiovascular intervention? A systematic review and meta-analysis of randomized controlled trials and observational studies. Medicine (Baltimore). 2015;94(44):e1910.

22. UK Prospective Diabetes Study Group. Tight blood pressure control and risk of macrovascular and microvascular complications in type 2 diabetes: UKPDS 38. BMJ. 1998;17(7160):703-13.

23. McEvoy JWCY, Rawlings A, Hoogeveen RC, Ballantyne CM, Blumenthal RS, Coresh J, Selvin E. 
Diastolic blood pressure, subclinical myocardial damage, and cardiac events: implications for blood pressure control. J Am Coll Cardiol. 2016;68(16):1713-22.

24. Vidal-Petiot E, Ford I, Greenlaw N, Ferrari R, Fox $\mathrm{KM}$, Tardif JC, et al. Cardiovascular event rates and mortality according to achieved systolic and diastolic blood pressure in patients with stable coronary artery disease: an international cohort study. Lancet. 2016;388(10056):2142-52.

25. Bronsert MR, Henderson WG, Valuck R, Hosokawa $\mathrm{P}$, Hammermeister K. Comparative effectiveness of antihypertensive therapeutic classes and treatment strategies in the initiation of therapy in primary care patients: a Distributed Ambulatory Research in Therapeutics Network (DARTNet) study. J Am Board Fam Med. 2013;26(5):529-38.

26. Terpstra WF, May JF, Smit AJ, Graeff PA, Havinga TK, van den Veur E, et al. Long-term effects of amlodipine and lisinopril on left ventricular mass and diastolic function in elderly, previously untreated hypertensive patients: the ELVERA trial. J Hypertens. 2001;19(2):303-9.

27. Muller-Brunotte R, Kahan T, Malmqvist K, Ring M, Edner M. Tissue velocity echocardiography shows early improvement in diastolic function with irbesartan and atenolol therapy in patients with hypertensive left ventricular hypertrophy: results form the Swedish Irbesartan Left Ventricular Hypertrophy Investigation vs Atenolol (SILVHIA). Am J Hypertension. 2006;19(9):927e36.

28. Yip GW, Wang M, Wang T, Chan S, Fung JW, Yeung L, et al. The Hong Kong diastolic heart failure study: a randomised controlled trial of diuretics, irbesartan and ramipril on quality of life, exercise capacity, left ventricular global and regional function in heart failure with a normal ejection fraction. Heart. 2008;94(5):573-80.

29. Ginelli P, Bella JN. Treatment of diastolic dysfunction in hypertension. Nutr Metab Cardiovasc Dis. 2012;22(8):613-8.

30. Massie BM, Carson PE, McMurray JJ, Komajda M, McKelvie R, Zile MR. Irbesartan in patients with heart failure and preserved ejection fraction. N Engl J Med. 2008;359(23):2456-67.

31. Yusuf S, Pfeffer MA, Swedberg K, Granger CB, Held $\mathrm{P}$, McMurray JJ, et al. Effects of candesartan in patients with chronic heart failure and preserved left-ventricular ejection fraction: the CHARM-Preserved Trial. Lancet. 2003;362(9386):777-81.

32. Setaro JF, Zaret BL, Schulman DS, Black HR, Soufer R. Usefulness of verapamil for congestive heart failure associated with abnormal left ventricular diastolic filling and normal left ventricular systolic performance. Am J Cardiol. 1990;66(12):981-6.

33. Mottram PM, Haluska B, Leano R, Cowley D, Stowasser M, Marwick TH. Effect of aldosterone antagonism on myocardial dysfunction in hypertensive patients with diastolic heart failure. Circulation. 2004;110(5):558-65.

34. Gradman AH, Basile JN, Carter BL, Bakris GL, Group ASoHW. Combination therapy in hypertension. J Am Soc Hypertens. 2010;4(1):42-50.

35. Jamerson K, Weber MA, Bakris GL, Dahlöf B, Pitt B, Shi V, et al. Benazepril plus amlodipine or hydrochlorothiazide for hypertension in high-risk patients. N Engl J Med. 2008;359(23):2417-28.

36. Chobanian AV, Bakris GL, Black HR, Cushman WC, Green LA, Izzo JL, et al. The Seventh Report of the Joint National Committee on Prevention, Detection, Evaluation, and Treatment of High Blood Pressure: the JNC 7 report. Hypertension. 2003;289(19):2560-72.

37. Calhoun DA, Lacourcière Y, Chiang YT, Glazer RD. Triple antihypertensive therapy with amlodipine, valsartan, and hydrochlorothiazide: a randomized clinical trial. Hypertension. 2009;54(1):32-9.

38. Mazza A, Lenti S, Schiavon L, Sacco AP, Dell'Avvocata F, Rigatelli $G$, et al. Fixed-dose triple combination of antihypertensive drugs improves blood pressure control: from clinical trials to clinical practice. Adv Ther. 2017;34(4):975-85.

39. Bangalore S, Kumar S, Lobach I, Messerli FH. Blood pressure targets in subjects with type 2 diabetes mellitus/impaired fasting glucose. Circulation. 2011;123(24):2799-810.

40. Cooper-DeHoff RM. Tight blood pressure control and cardiovascular outcomes among hypertensive patients with diabetes and coronary artery disease. JAMA. 2010;304(1):61-8. 